\title{
A Taxonomy of Passive Behaviors in People with Alzheimer's Disease
}

\section{Kathleen Byrne Colling}

Purpose: To construct a taxonomy of passive behaviors for understanding people with Alzheimer's disease. Passive behaviors are those associated with decreased motor movements, decreasing interactions with the environment, and feelings of apathy and listlessness. Little is known about behaviors associated with passivity, and these behaviors have not been categorized.

O rganizing Construct: Taxonomy construction. Passive behaviors in people with A Izheimer's disease were conceptualized as disturbing behaviors, patterns of personality change, and negative symptoms.

Methods: The taxonomy was devel oped using critical reviews of 15 empirical studies published 1985 through 1998. Procedures included listing behaviors; clustering behaviors into inductively derived groupings; conducting an expert panel-review, making revisions, and conduting a second review; establishing global and category-by-category reliability using Cohen's Kappa.

Findings: Thenonhierarchic, natural taxonomy indicated five categories of behaviors associated with passivity in Alzheimer's disease: diminutions of cognition, psychomotor activity, emotions, interactions with people, and interactions with the environment. Analysis indicated substantial agreement beyond chance and show ed statistically significant agreement among the six nurse-expert raters. A reas of synchrony between the taxonomy and the $\mathrm{N}$ eed-D riven D ementia Compromised Behavior Model were identified.

Conclusions: This taxonomy of passive behaviors in patients with A Izheimer's disease show ed empirical rigor and compatibility with a middle-range theory and can be viewed as a sensitizing analytic scheme to guide future practice, research, and theory development.

Journal of Nursing SaholarSHip, 2000; 32:3, 239-244. @2000 Sigma Theta Tau InItranational.

[Key words: taxonomy, passivity, Alzheimer's D isease]

A pathy, withdrawal, reduced initiative, and diminished affect constitute essential characteristics associated with dementia of the Alzheimer's type (DAT) (Galynker, Roane, M iner, Feinberg, \& Watts, 1995). However, overtly disturbing behaviors such as agitation, (Gerdner \& Buckwalter, 1994), aggression (W hall, Gillis, Yankou, Booth, \& Beel-Bates, 1992), wandering (Algase, 1992), and problematic vocalizations (Burgio et al., 1994), have received considerable attention in the literature. Recent research has indicated that passive behaviors affect $61 \%$ to $88 \%$ of people with DAT (Galynker et al., 1995). These behaviors are associated with cognitive decline (Kurita, Blass, Nolan, Black, \& Thaller, 1993), occur on a daily basis, and are most often reported as being unresponsive to intervention (Everitt, Fields, Soumerai, \& Avorn, 1991). Furthermore, passive behaviors correlate with the severity of dementia and exist despite treatment with medication, comorbid systemic illness, and an absence of depressive symptoms (R eichman, Coyne, A mirneni, M olino, \& Egan, 1996).

Three distinct but related approaches to conceptualizing the constellation of passive behaviors associated with DAT have emerged from initial research efforts. First, Kolanowski
(1995) used concept synthesis methods (Walker \& Avant, 1995) to identify passivity as one of five disturbing behaviors characteristic of dementia. In addition to aggressive motor behavior, nonaggressive psychomotor behavior, verbally aggressive behavior, and functionally impaired behavior, the category of passive behavior was identified. Passive behaviors were defined as those which were a diminution of behavior, that is, a decrease in gross motor movement accompanied by apathy and lack of interaction with the environment (Kolanowski, 1995). A second approach has centered on identifying apathy and passivity as distinct patterns of personality changes that occur as the disease progresses (Chatterjee, Strauss, Smyth, \& Whitehouse, 1992;

Kathleen Byrne Colling, RN, PhD, Rho, Assistant Research Scientist, University of Michigan, School of Nursing, Ann Arbor, Ml. The author was supported by a postdoctoral fellowship National Science Research Award. The author acknowledges the wisdom and support of Ann Whall, RN, PhD, FAAN, Barbara Therrien, $\mathrm{RN}, \mathrm{PhD}$, FAAN, and DonnaAlgase, $\mathrm{RN}, \mathrm{PhD}$, in conducting the research and manuscript preparation. Correspondence to Dr. Colling, University of Michigan School of Nursing, 400 North Ingalls, Ann Arbor, Ml 48109-0482. Email: kcolling@umich.edu

Accepted for publication October 13, 1998. 
Kolanowski, Strand, \& Whall, 1997; Petry, Cummings, Hill, \& Shapira 1988, 1989; and Rubin, M orris, \& Berg, 1987). A third approach by researchers has led to the viewpoint that passivity, withdrawal, and apathy may be considered elements of a "negative symptom" complex analogous to the state described in schizophrenia (Doody, M assman, M ahurin, \& Law, 1995; Galynker et al., 1995; and R eichman \& Coyne, 1993).

Beyond these initial research efforts, a need existed to analyze the current know ledge base to identify the theoretical and methodological problems in describing and understanding the nature and meaning of these behaviors. The first step was a review and critique of 15 research studies of behavioral symptoms associated with passivity in DAT (Colling, 1999a). The investigation showed disagreement among researchers about specific descriptors or constructs to define the phenomenon. No established operational definition unified these behaviorial definitions into a syndrome that could be measured and correlated with other variables (R eichman \& Coyne, 1993). The need to classify passive behaviors into well-d efined entities was necessary for theory development, clinical applications, and measurement because no instrument has been specifically designed to measure passive behaviors in dementia (C olling, 1999a).

This article describes the use of concept synthesis (Walker \& Avant, 1995) for extracting meaningful categories and behaviors from 15 empirical studies identified as key to the investigation of passive behaviors. This method was selected to guide the investigator because the body of knowledge about passivity embodied little or no conceptual development, and concepts that had been named had no effect on theory or practice. Three approaches to concept synthesis include qualitative, quantitative, and literary. The literary approach was chosen to guide this investigation. With this approach, the literature about the phenomenon can be examined for the purpose of acquiring new insights and the literature itself becomes the database (Walker \& Avant, 1995). From this investigation, a taxonomy was derived to organize the concepts and link them according to specific criteria (R asch, 1987).

\section{Methods}

The empirical studies on passive behaviors in DAT were identified through computerized searches of $M$ edline ( $N$ ational Library of $M$ edicine), PsychL it (Psychological A bstracts), and CIN A H L (Cumulative Index of N ursing and Allied $\mathrm{H}$ ealth Literature) for the period from 1985 to 1998. Key words in the search were: Alzheimer's, dementia, apathy, behavior, passive symptoms, and negative symptoms. M anual searches of tables of contents of current nursing and interdisciplinary journals to find publications, which had not been indexed on computer databases, were also done. The ancestry method was used to trace citations from reference lists of studies. Criteria for selection included the following: (a) data-based research; (b) a focus on concepts associated with passivity in DAT, such as apathy, withdrawal, and disinterest; and (c) publication in refereed journals. A total of 15 studies published from 1987 to 1997 were chosen. These included the works of the following investigators: Burns, Folstein, Brandt and Folstein (1990); Chatterjee, Strauss, Smyth, and W hitehouse (1992); Doody, M assman, M ahurin, and Law (1995); Everitt, Fields, Soumerai, and Avorn (1991); Galynker, R oane, M iner, Feinberg, and Watts (1995); Gilley, Wilson, Bennett, Bernard, and Fox (1991); H aight and Warren (1991); Kolanowski, Strand, and Whall (1997); O tt, Tate, Gordon, and Heindel (1996); Petry, Cummungs, Hill, and Shapira (1988, 1989); Reichman, Coyne, A mirneni, M olino, and Egan (1996); Rubin, M orris, and Berg (1987); Strauss, Pasupathi, and Chatterjee (1993); and Teri, Borson, Kiyak, and Yamagishi (1989).

Twelve of the 15 studies did not include a theoretical or conceptual framew ork. O perational definitions of variables, that were narrow in focus and could be manipulated, were included; however, these definitions varied widely among studies. Studies were conducted predominantly in community settings with a variety of instruments, data-collection methods, age groups, and methods (Colling, 1999a). Despite limitations of the studies, behaviors associated with passivity showed similarities among studies.

The next step in concept synthesis was to formulate a taxonomy, or category coding system, as represented in the work of Ryan-Wenger (1992). Taxonomy development is a theoretical operation in which groups, classes, or sets are systematically organized and linked according to some criterion. A taxonomy may be artificial or natural, hierarchic or nonhierarchic, and may be derived inductively or deductively. Because taxonomy development includes systematically organizing concepts and criterion links, its construction may be considered a conceptual framework (Rasch, 1987).

The first step in constructing this taxonomy was to list all the behaviors ( $\mathrm{N}=82$ ) that had been identified by the authors of the 15 empirical studies related to passive behavior in DAT. The criterion for retaining behavioral items was Kolanowski's (1995) definition of passive behavior in peoplewith dementia. An attempt was also made to include as many behaviors as possible so to avoid eliminating any terms prematurely. The next step was "clustering," or identifying behaviors that seemed closely related (Walker \& Avant, 1995). These groupings were inductively derived. No preconceived categories were used and no attempt was made to infer the specific motivation causing the behavior (Ryan-Wenger, 1992). Six groupings weretitled: (a) diminution of cognition, (b) diminution of psychomotor activity, (c) diminution in feeling emotions, (d) diminution of responding to emotions, (e) diminution of interactions with people, and (f) diminution of interactions with the environment.

$\mathrm{N}$ ext, an instrument was constructed which included definitions for each of the six categories of passive behaviors. The definitions were determined after review of the literature, examination of potential clusterings of the behavioral items, and consideration of the most accurate descriptors of each 
category. A panel of six doctorally prepared nurse scientists with clinical and research experience in gerontology was instructed to place each behavioral description in the one category the rater deemed most appropriate. A sheet for comments allowed the raters to list specific items with which they had difficulty, to explain why, and to suggest revisions of the categories together with their rationale for the change.

Multiple-rater kappa was calculated according to the method of Siegel (1988), initially published in Fleiss (1971). A kappa of 0 indicates that observed agreement among raters is equal to agreement caused by chance alone. A kappa of 1.0 indicates perfect agreement among raters, beyond what would be expected by chance. The multiple-rater kappa for the taxonomy was 0.42 , indicating "fair" agreement among raters according to Landis and Koch (1977) and "good" agreement according to Fleiss (1981).

N ext, Fleiss' (1971) formula, for the extent of agreement among the six raters for specific items in the taxonomy, was calculated. All items with extent of agreement scores at or above 0.50 , or those placed in a specific category by a majority of the raters, were retained. Any redundant or ambiguous wording was then clarified. Categories 3 and 4 , the "feeling of" and "responding to" emotion categories, were combined into a single category titled "diminution of emotions." Combining two or more categories into one has been recommended to improve reliability of a coding system (Garvin, Kennedy, \& Cissna, 1988). This method was used because raters expressed difficulty distinguishing "feeling emotion" from "responding to emotion." The category titled "Diminution of Emotions" was defined as the decrease or absence of the ability to experience or respond to human emotions. The reduced and revised taxonomy contained 63 items which appeared more likely to fall clearly into one of the category groups.

The next step was a second round of independent ratings by the same six experts on the panel. The overall multiplerater kappa was 0.61, indicating fair to good (Fleiss, 1981) or substantial agreement (Landis \& Koch, 1977) beyond chance. An item-by-item analysis was conducted using extentof-agreement scores, resulting in deletion of eight additional items, and keeping 54-items. Items were retained in this version of the taxonomy if extent of agreement for a particular item was at or above 0.50 , or if more than half (four) of the raters placed an item in the same category. The overall kappa, recalculated for the 54-item taxonomy, improved to 0.698 , indicating fair to good (Fleiss, 1981) or substantial agreement (Landis \& Koch, 1977) beyond chance. This kappa also showed statistically significant agreement among raters $(p<.001)$.

Additional statistics were calculated to assess reliability of the rating process. The investigator used SPSS to calculate Cohen's kappa for the final version of the overall taxonomy, for all possible pairs of raters, a total of 15 comparisons. These kappas ranged from 0.60 , indicating fair or good (Fleiss, 1971) or moderate agreement (Landis \& Koch, 1977), to 0.83 , indicating strong (Fleiss, 1971) or almost perfect agreement (Landis \& Koch, 1977). Strength of agreement among all raters for each category of the taxonomy was also calculated according to Fleiss's (1971) formula for agreement on a particular category. The extent-of-agreement kappas for the five categories are listed in Table 1. These range from a high of 0.81 for Category 3 "Diminution of Emotions" indicating strong (Fleiss, 1971) or almost perfect agreement (Landis \& Koch, 1977) to a low of 0.55 for category 2 of "Diminution of Psychomotor Activity" indicating fair or good (Fleiss, 1971) or moderate agreement (Landis \& Koch, 1977).

\section{Results}

The taxonomy which resulted (Table 2 ) is nonhierarchic, because it uses one attribute or one set of attributes to group people, and it is not arranged into classes that are increasingly inclusive (R asch, 1987). The order of the categories was kept consistent through both sets of reviews by the expert panel and statistical analyses were also performed in the same order.

The taxonomy is considered natural instead of artificial because the passive behaviors were grouped under headings that were fundamental to people with DAT (Rasch, 1987). Behavioral items such as "Iess enthusiastic," "less affectionate," and "less cheerful than usual" were included in Category 3, "Diminution of Emotions" to indicate these observable characteristics.

Table 1. Taxonomy of Passivity in Patients With Alzheimer's Disease: Categories, Definitions, and Extent-of-Agreement Kappas of the Category Coding System

Category
1. Diminution of cognition
2. Diminution of psychomotor
activity

Definitions Kappa

Lessening of mental processes associated with thinking and knowing.

Diminution of psychomotor

Decrease in the spontaneous and purposeful performance of voluntary motor movements.

3. Diminution of emotions

Decrease or absence of the ability to experience or respond to human emotions.

4. Diminution of interactions with people

Lessening of behaviors indicative of or necessary to acting upon or with another or others.

5. Diminution of interactions with the environment

Lessening of behaviors indicative of or necessary to acting upon or with the physical surroundings. 0.73

(1)


Table 2. Taxonomy of Passive Behaviors

Category and definition

1. Diminution of Cognition: Lessening of mental processes associated with thinking and knowing.

2. Diminution of psychomotor activity: Decrease in the spontaneous and purposeful performance of voluntary motor movements.

3. Diminution of emotions:

Decrease or absence of the ability to experience human emotions, respond to human emotions, or both.

4. Diminution of interactions with people: Lessening of behaviors indicative of or necessary to acting upon or with another or others.

5. Diminution of interactions with the environment: Lessening of behaviors indicative of or necessary to acting upon or with the physical surroundings.
1. Demonstrates less self reliance

2. Displays diminished initiative

3. Decreased ability to be goal-directed

4. Decrease in expression of thought through speech

1. Sits quietly with a decrease in spontaneous movements

2. Fewer gestures to express emotions

3. Seems physically inert and "does nothing"

4. Decrease in the performance of activities of daily living

1. Unchanging "wooden" and "frozen" facial expression

2. Inability to feel intimacy and closeness

3. Decrease in sexual feelings

4. Failure to smile or laugh when prompted

5. Fewer vocal inflections to express emotions

6. Less enthusiastic

7. Less affectionate

8. Diminished emotional responsiveness

9. Less cheerful than usual

1. Decreased interest in spending time with friends

2. Poor eye contact with others

3. Decrease in sexual activity with significant other

4. Less generous with others

5. Less responsive when interacting with others

6. Displays disinterest to others

7. Seems "disconnected" from others

1. Decrease in recreational activities or hobbies

2. Seems to be withdrawing from activities

3. Seems unmoved by the environment

4. Lack of interaction with the environment
Behaviors

5. Decrease in ability to imagine

6. Demonstrates less intellectual curiosity

7. Demonstrates a decrease in conscientiousness

8. Decrease in ability to reexamine social, political, and religious values

5. Slowness of movements

6. Decrease of behaviors

7. Decrease in gross motor movements

8. Demonstrates underactivity

10. Demonstrates a flat affect

11. Emotional "death"

12. Demonstrates "blunting" of emotions

13. Lacks feelings of desire

14. Seems apathetic

15. Gets or feels no pleasure

16. Withdraws emotionally

17. Less receptive to inner feelings

18. Endures emotionally rather than protesting unpleasant happenings

8. Uninvolved with people

9. Absence of social risk-taking behavior

10. Withdraws from others

11. Has fewer close ties with others

12. Does not prefer the company of others

13. Tends to be submissive when interacting with others

5. Avoids a stimulating environment

6. Less open to a variety of experiences

7. Less willing to try different activities

\section{Evaluation of the Taxonomy}

Empirical. Ryan-Wenger (1992) said that the validity of findings can be ascertained by verification that similar behaviors appeared in more than one study despite different samples and data-collection methods. Although passive behaviors may have been conceptualized differently, for example, as negative behaviors or personality changes, similar behaviors such as apathy were reported. In addition, the final taxonomy contains five categories and 54 items indicating that the domain of possible behaviors is well represented. Category 5, "Diminution of Interactions with the Environment," contains the smallest number of behavioral items ( $n=7)$; perhaps future research will indicate more behaviors in this category. Both global reliability, or the extent to which coders could consistently use the entire coding system across all categories, and category-by-category reliability demonstrated that common labels were consistently applied to each identified category by the panel of expert raters.

Theoretical. Turner (1986) suggested that the development of analytical schemes is important and necessary for theorizing to sensitize researchers and guide theory development without being rigid and inflexible. Although this taxonomy of passive behaviors has heuristic value, human behavior is complex and may not always be compatible with separation into distinct components (J acox \& Webster, 1997).

This limitation exists in constructing any taxonomy of behavioral phenomena such as passivity. However, the identification of behaviors specific to a phenomenon is a prerequisite for empirical investigation. In this case, the categories and behaviors were identified through constructing the taxonomy and were used to design the Passivity in Dementia Scale (PDS), a research questionnaire which is currently undergoing preliminary psychometric testing. 
An important consideration in evaluating the taxonomy relates to its theoretical structure and meanings. Reynolds (1971) recommended that a taxonomy be consistent with concepts used in the statements that indicate other purposes of science. This tenet is consistent with Walker and Avant's (1995) final step in the concept synthesis process, that of determining, if possible, how the new concept fits into existing theory and acknowledging any new insights and new approaches to research and practice.

A reas of synchrony are evident between the taxonomy and the mid-range theoretical propositions of the $\mathrm{N}$ eedDriven Dementia-Compromised Behavior (N DB) framework (Algase et al., 1996). This model indicates that behaviors of people with cognitive impairment arise from pursuit of goals or as expression of needs. These behaviors show the interaction of background and proximal factors that are found within either the person, the environment, or both. Background factors include past personality, gender, education, and occupation. Proximal factors are characterized as physiological and psychological need states and the physical and social environments. These factors are thought to precipitate disturbing behaviors in dementia. Using the NDB model, passive behaviors by people with cognitive impairment would be viewed as representing the most integrated and meaningful response possible given limitations of the disease, the person, and the environment.

The headings and categorized behaviors of the taxonomy can be helpful in further development and testing of the NDB model. The definition and categorization of passive behaviors gives credence to the argument that passive behavior should be added to aggression, problematic vocalizations, and wandering as another disturbing behavior by people with dementia. Variables within the categories of the N DB model also seem to be related to categories of the taxonomy. For example, the NDB model's psychosocial background variables can be thought to result in behaviors identified in categories two, three, four, and five of the taxonomy. These categories illustrate passivity as a lessening of psychomotor activity, emotions, interactions with people and interactions with the environment. In addition, clinical application of the N D B model to people with dementia who demonstrate passive behaviors has been explored by describing how the variables can be applied in clinical practice (Colling, 1999b).

The identification of the specific background and proximal factors within the NDB model thought to be specific to passivity and the extent to which these factors allow prediction of passivity are research questions that can be addressed using the differentiating categories within the taxonomy. The possibility also exists that additional behaviors, not yet identified, will have implications for future theoretical investigation of both the NDB model and the taxonomy. With continued development, the taxonomy should meet Reynolds (1971) criteria of containing categories that are mutually exclusive, exhaustive, and consistent with the concepts which help researchers describe, explain, and predict the phenomenon.

\section{Conclusions}

This taxonomy of passive behaviors in Alzheimer's disease has been subjected to measures of validity and reliability. It is compatible with a middle-range theory, that is, the $\mathrm{N}$ eedDriven Dementia-Compromised Behavior Model. A more specific micromodel of passivity might be needed to assess behaviors more precisely. The taxonomy has shown empirical rigor and can be used as an analytical scheme in future research, practice, and theory development. JNs

\section{References}

Algase, D.L. (1992). Cognitive discriminants of wandering among nursing home residents. $\mathbf{N}$ ursing $\mathbf{R}$ esearch, 41(2), 78-81.

Algase, D.L., Beck, C., Kolanowski, A., Whall, A., Berent, S.K. Richards, K., \& Beattie, E. (1996). N eed-Driven dementia-compromised behavior: An alternative view of disruptive behavior. American Journal of Alzheimer's D isease, 11(6), 10-19.

Burns, A., Folstein, S., Brandt, J., \& Folstein, M . (1990). Clinical assessment of irritability, aggression and apathy in $\mathrm{H}$ untington and $\mathrm{A}$ Izheimer disease. The Journal of $\mathrm{N}$ ervous and $\mathrm{M}$ ental Disease, 178(1), 20-26.

Chatterjee, A., Strauss, M.E., Smyth, K.A., \& Whitehouse, P.J. (1992). Personality changes in Alzheimer's disease. Archives of $\mathbf{N}$ eurology, 49, 486-491.

Colling, K.B. (1999 a). Passive behaviors in Alzheimer's disease: A descriptive analysis. American Journal of Alzheimer's D isease, 14(1), 27-40.

Colling, K.B. (1999 b). Passive behaviors in dementia; Clinical applications of the need-driven dementia-compromised behavior model. Journal of Gerontological N ursing, 25(9), 27-32.

Doody, R.S., M assman, P., M ahurin, R., \& Law, S. (1995). Positive and negative neuropsychiatric features in Alzheimer's disease. Journal of N europsychiatry, 7(1), 54-60.

Everitt, D.E., Fields, D.R., Soumerai, S.S., \& Avorn, J. (1991). Resident behavior and staff distress in the nursing home. Journal of the American G eriatrics Society, 39(8), 792-798.

Fleiss, J.L. (1971). M easuring nominal scale agreement among many raters. Psychological Bulletin, 76(5), 378-382.

Fleiss, J.L. (1981). Statistical methods for rates and proportions (2nd ed.). N ew York: Wiley.

Galynker, I.I., R oane, D.M ., M iner, C .R ., Feinberg, T.E., \& Watts, P. (1995). $\mathrm{N}$ egative symptoms in patients with Alzheimer's disease. The American Journal of G eriatric Psychiatry, 3(1), 52-59.

G arvin, B.J., Kennedy, C.W., \& Cissna, K.N . (1988). R eliability in category coding systems. N ursing R esearch, 37(1), 52-55.

Gerdner, L.A., \& Buckwalter, K.C. (1994). A nursing challenge: A ssessment and management of agitation in Alzheimer's patients. Journal of Gerontological N ursing, 20(4), 11-20.

Gilley, D.W., Wilson, R.S., Bennett, D.A., Bernard, B.A., \& Fox, J.H . (1991). Predictors of behavioral disturbance in Alzheimer's disease. Journal of Gerontology: Psychological Sciences, 46(6), 362-371.

H aight, B.K., \& Warren, J. (1991). A pathy: Development of a nursing diagnosis. Applied N ursing Research, 4(4), 186-188.

Jacox, A.K., \& Webster, G . (1997). Competing theories of science. In L.H. $\mathrm{N}$ icoll (Ed.), Perspectives on nursing theory (423-431). Philadelphia: Lippincott.

Kolanowski, A.M. (1995). Disturbing behaviors in demented elders: A concept synthesis. Archives of Psychiatric N ursing, 9(4), 188-194.

Kolanowski, A.M., Strand, G., \& Whall, A. (1997). A pilot study of the relation of premorbid characteristics to behavior in dementia. Journal of Gerontological N ursing, 23(2), 21-30.

Kurita, A., Blass, J.P., N olan, K.A., Black, R.S., \& T haller, H.T. (1993). Relationship between cognitive status and behavioral symptoms in Alzheimer's disease and mixed dementia. Journal of the American G eriatrics Society, 41(7), 732-736. 
Landis, J.R ., \& Koch, G.G . (1977). The measurement of observer agreement for categorical data. Biometrics, 33(1), 159-174.

Ott, B.R., Tate, C.A., Gordon, N.M., \& Heindel, W.C. (1996). Gender differences in the behavioral manifestations of A lzheimer's disease. Journal of the American G eriatrics Society, 44, 583-587.

Petry, S., Cummings, J.L., H ill, M .A., \& Shapira, J. (1988). Personality alterations in dementia of the Alzheimer type. Archives of N eurology, 45, 1187-1191.

Petry, S., C ummings, J.L., H ill, M .A., \& Shapira, J. (1989). Personality alterations in dementia of the Alzheimer type: $\mathrm{A}$ three-year follow-up study. Journal of G eriatric Psychiatry and N eurology, 2(4), 203-207.

R asch, R.F.R. (1987). The nature of taxonomy. Image: Journal of $\mathbf{N}$ ursing Scholarship, 19(3), 147-149.

Reichman, W.E., \& C oyne, A.C. (1993). N egative symptoms in Alzheimer's disease (abstract). American Journal of Psychiatry, 1(3), 262.

Reichman, W.E., C oyne, A.C., A mirneni, S., M olino, B., \& Egan, S. (1996). $\mathrm{N}$ egative symptoms in Alzheimer's disease. American Journal of Psychiatry, 153(3), 424-426.

Reynolds, P.D. (1971). A primer in theory construction. N ew York: BobbsM errill.
R ubin, E.H ., M orris, J.C ., \& Berg, L. (1987). The progression of personality changes in senile dementia of the Alzheimer's type. Journal of the American G eriatric Society, 35, 721-725.

Ryan-Wenger, N.M . (1992). A taxonomy of children's coping strategies: A step toward theory development. Journal of 0 rthopsychiatry, 62(2), 256263.

Strauss, M .E., Pasupathi, M ., \& C hatterjee, A. (1993). Concordance between observers in descriptions of personality change in Alzheimer's disease. Psychology and Aging, 8(4), 475-480.

Teri, L., Borson, S., Kiyak, A., \& Yamagishi, M. (1989). Behavioral disturbance cognitive dysfunction, and functional skill: Prevalence and relationship in Alzheimer's disease. Journal of the American Geriatrics Society, 37, 109-116.

Turner, J.H . (1986). The structure of sociological theory (4th ed.). Chicago: Dorsey Press.

Walker, L.O., \& Avant, K.C. (1995). Strategies for theory construction in nursing (3rd ed.). N orwalk, CT: A ppleton \& Lange.

Whall, A.L., Gillis, G.L., Yankou, D., Booth, D.E., \& Beel-Bates, C.A. (1992). Disruptive behavior in elderly nursing home residents: A survey of nursing staff. Journal of G erontological N ursing, 18(10), 13-17. 